\title{
Two-layer inverse model for improved longitudinal preclinical tumor imaging in the spatial frequency domain
}

Syeda Tabassum

Vivian Pera

Gage Greening

Timothy J. Muldoon

Darren Roblyer 


\title{
Two-layer inverse model for improved longitudinal preclinical tumor imaging in the spatial frequency domain
}

\author{
Syeda Tabassum, ${ }^{a}$ Vivian Pera, ${ }^{b}$ Gage Greening, ${ }^{c}$ Timothy J. Muldoon, ${ }^{c}$ and Darren Roblyer ${ }^{b, *}$ \\ aBoston University, Department of Electrical and Computer Engineering, Boston, Massachusetts, United States \\ ${ }^{\mathrm{b} B o s t o n}$ University, Department of Biomedical Engineering, Boston, Massachusetts, United States \\ 'University of Arkansas, Department of Biomedical Engineering, Fayetteville, Arkansas, United States
}

\begin{abstract}
Spatial frequency domain imaging (SFDI) is a widefield, noncontact, and label-free imaging modality that is currently being explored as a new tool for longitudinal tracking of cancer therapies in the preclinical setting. We describe a two-layer look-up-table (LUT) inversion algorithm for SFDI that better accounts for the skin (top layer) and tumor (bottom layer) tissue geometry in subcutaneous tumor models. Monte Carlo (MC) simulations were conducted natively in the spatial frequency domain, avoiding discretization errors associated with Fourier or Hankel transforms of conventional MC simulation results. The two-layer LUT was validated using two-layer tissue mimicking optical phantoms, in which the optical property extractions of the bottom (tumor) layer were determined to be within $20 \%$ and $11 \%$ of the true values for $\mu_{a}$ and $\mu_{s}^{\prime}$, respectively. A sensitivity analysis was conducted to evaluate how imperfect top layer estimates affect bottom-layer optical property extractions. Finally, the two-layer LUT was used to reanalyze a prior longitudinal data set, which revealed larger therapy-induced changes in optical scattering and a more hypoxic tumor environment compared to the homogeneous LUT. The two-layer LUT described here improves the accuracy of subcutaneous tumor imaging, and the general methodology can be applied for arbitrary multilayer SFDI applications. ๑ 2018 Society of Photo-Optical Instrumentation Engineers (SPIE) [DOI: 10.1117/1.JBO.23.7.076011]
\end{abstract}

Keywords: spatial frequency domain; diffuse optics; oncology; photon migration; inversion algorithm; optical properties.

Paper 180215R received Apr. 11, 2018; accepted for publication Jul. 9, 2018; published online Jul. 27, 2018.

\section{Introduction}

Despite an ever growing array of treatment options for patients with solid tumors, including chemotherapies, targeted drugs, and immunotherapies, individual patient response remains highly variable. ${ }^{1}$ Furthermore, most patients who die from cancer will develop resistance to the drugs they are given. ${ }^{1}$ Surveillance of the in vivo tumor state with methods that can detect both treatment response and resistance would provide opportunities for physicians to dynamically adapt and personalize regimens to maximize overall treatment efficacy. Unfortunately, current standard of care imaging tools (e.g., MRI and PET) are limited for this type of frequent longitudinal monitoring due in part to the high expense, lack of portability, safety concerns, and in some cases, patient discomfort during measurements. ${ }^{2-4}$ There is an interest in exploring the use of clinical diffuse optical imaging (DOI) to meet this need as these techniques provide a metabolic and molecular profile of tumors while being label-free, safe, and generally low cost. ${ }^{5}$ For example, over the last decade multiple studies have used DOI tools to track optical changes in breast cancer patients receiving neoadjuvant chemotherapy. ${ }^{6-8}$ These studies are largely consistent in their findings that DOI imaging methods reveal changes in optical absorption, scattering, as well as hemoglobin, water, and lipid content that correlate strongly with treatment response determined by pathology.
While encouraging, there are open questions related to how DOI parameters manifest for different systemic therapies, drug combinations, and dosing schedules, as well as how DOI parameters correlate with underlying tumor and host biology at the cellular and molecular levels. Although there have been some prior efforts to correlate in vivo DOI clinical measurements with tissue samples obtained by biopsy or from surgical specimens, ${ }^{9,10}$ obtaining tissue samples during treatment remains a challenge. Alternatively, the preclinical setting provides opportunities for testing new or emerging therapeutic strategies while simultaneously allowing better access to tissues for ex vivo analysis, potentially providing a pathway for placing DOI metrics in a biological context for a variety of treatment strategies. For example, we have shown in a previous study that spatial frequency domain imaging (SFDI) can be used to monitor the in vivo tumor state of a subcutaneous tumor xenograft model grown in mouse over a period of 45 days. ${ }^{11}$ SFDI is a widefield DOI modality, capable of tracking the same noninvasive and label-free metrics measured using clinical DOI modalities and is well suited for preclinical oncology work given its shallower penetration depth (typically $\mathrm{mm}$ ). ${ }^{11,12}$

Much of the prior published works using SFDI for both small animal and clinical research have utilized the assumption of homogeneity in depth when extracting optical properties from tissue. ${ }^{13-16}$ For example, in our prior preclinical monitoring study, we modeled mouse tumor tissue as a semi-infinite 
homogeneous medium and used the results of a Monte Carlo (MC) simulation to create a look-up-table (LUT) inversion algorithm to recover optical property estimates from SFDI measurements of diffuse reflectance. ${ }^{11}$ However, tissue geometry is complex, and in the case of subcutaneous tumors in mice, a thin skin layer is located above the tumor, which remains unaccounted for in a homogeneous model. Although there are several prior studies that have described inversion algorithms that utilize a layered-tissue structure ${ }^{17-21}$ or utilize tomographic reconstructions with SFDI, ${ }^{22,23}$ we describe, in this work, the first use of MC simulations conducted natively in the spatial frequency domain to make a two-layer LUT inversion algorithm that closely matches the true physiology and optical characteristics of preclinical tumor models.

The organization of this manuscript is as follows: First, we describe the MC simulation parameters and methods used to generate a two-layer LUT. We then compare its performance to two different homogeneous LUTs. We then validate the two-layer LUT algorithm using experimental measurements made on custom two-layer tissue-simulating phantoms. Next, we conduct a sensitivity analysis to determine how sensitive the two-layer LUT is to imperfect knowledge of the top (skin) layer parameters, including optical properties and layer thickness. Then we utilize the two-layer LUT to reanalyze an in vivo data set from a previously published longitudinal treatment monitoring study and compare the results to those obtained with a homogeneous LUT. ${ }^{11}$ Finally, we conclude by discussing the advantages and limitations of the two-layer methodology and its potential for future applications in preclinical oncology.

\section{Materials and Methods}

\subsection{Spatial Frequency Domain Imaging}

SFDI is a widefield DOI method that provides pixel-level extractions of optical properties. SFDI instrumentation, data acquisition, processing, and analysis procedures have been described in detail in the previous publications. ${ }^{11,12}$ Briefly, SFDI utilizes projections of spatially modulated light onto a phantom or tissue surface, and the remitted diffuse light is collected with a CCD camera. Images are typically collected at multiple wavelength bands and at least two spatial frequencies. A demodulation algorithm is used to extract the AC tissue response from each spatial frequency. Demodulated image data are then calibrated to remove the instrument response using measurements from a tissue-simulating phantom with known optical properties. Corrections for height and angle of the object surface may also be employed for nonflat surfaces. ${ }^{24}$ Diffuse reflectance $\left(R_{d}\right)$ maps acquired at a minimum of at least two spatial frequencies $\left(f_{x}\right)$ are then used as inputs to an inverse model to extract tissue absorption $\left(\mu_{a}\right)$ and reduced scattering $\left(\mu_{s}^{\prime}\right)$ as a function of wavelength. ${ }^{12,25}$

In this study, the OxImager RS SFDI system (Modulated Imaging, Inc., Irvine, California) was used for all measurements. The system has an imaging field of view of $15 \times 20 \mathrm{~cm}$, and for this study illumination wavelengths of $659,691,731$, and $851 \mathrm{~nm}$ and spatial frequencies of 0 and $0.1 \mathrm{~mm}^{-1}$ were utilized. The sample was illuminated with one-dimensional sinusoidal spatial patterns, and each $f_{x}$ was projected at three offset spatial phases $(0,120$, and $240 \mathrm{deg})$. Demodulation was performed using previously described methods, specifically Eq. (20) in Cuccia et al. ${ }^{12}$ An MC-based two-frequency LUT inversion algorithm was used to map $R_{d}$ values to optical properties (described in more detail in Sec. 2.2). For tissue measurements, the four $\mu_{a}$ values extracted at the four illumination wavelengths were used to compute tissue-level chromophore concentrations using Beer's Law. Oxyhemoglobin $\left(\mathrm{HbO}_{2}\right)(\mu \mathrm{M})$, deoxyhemoglobin $(\mathrm{Hb})(\mu \mathrm{M})$, total hemoglobin $(\mathrm{THb})(\mu \mathrm{M})$, and oxygen saturation $\left[\mathrm{StO}_{2}(\%): \mathrm{HbO}_{2} / \mathrm{THb} \times 100\right]$ were extracted from mouse tissue measurements. ${ }^{5,11}$ Scattering amplitude and scattering slope were also computed by fitting the wavelengthdependent $\mu_{s}^{\prime}$ values to a power law on a pixel by pixel basis. ${ }^{11}$

\subsection{Monte Carlo Simulations for the Generation of LUT-Based Inverse Models}

\subsubsection{Prior work and the implementation of the Gardner method for estimating $R_{d}$ in the spatial frequency domain}

Several prior publications from our group and others have utilized a two-frequency LUT inversion algorithm to extract optical properties from SFDI-derived $R_{d}$ values. ${ }^{11,24,26,27}$ At the core of this LUT algorithm is a single conventional MC simulation for a semi-infinite homogeneous medium. The simulation results are postprocessed to provide $R_{d}$ values for arbitrary $\mu_{a}$ and $\mu_{s}^{\prime}$ combinations using the methods described in Martinelli et al. ${ }^{28}$ A discrete Hankel transform is then used to transform the spatially resolved $R_{d}$ values to the spatial frequency domain. An LUT is constructed by scaling (i.e., postprocessing) the MC results for a desired range and step size of $\mu_{a}$ and $\mu_{s}^{\prime}$ values. In our work, a linear interpolation method is then implemented using MATLAB's "griddata" function, whose input is measured $R_{d}$ values and the LUT, and whose output is best fit optical properties.

This method has several limitations. First, the scaling method in Martinelli et al. ${ }^{28}$ is only described for homogeneous media, thus making it currently inapplicable for modeling multilayer tissue geometries. Additionally, the discrete Hankel transform is sensitive to discretization errors, which may lead to inaccuracies and artifacts depending on the density of the spatial sampling used. ${ }^{29}$

A method for obtaining multilayer MC results in the spatial frequency domain was recently described by Gardner and Venugopalan. ${ }^{29}$ The Gardner method obtains $R_{d}$ estimates natively in the spatial frequency domain and is, therefore, not subject to the same discretization errors caused by taking a discrete transform of spatially resolved $R_{d}$. The Gardner method computes a frequency-dependent photon weight, as shown in Eq. (1), for a two-layer medium and a spatially modulated source in the $x$-direction

$W_{n}=\exp \left(-\mu_{a, 1} d_{1, n}-\mu_{a, 2} d_{2, n}\right) \exp \left(-2 \pi i f_{x} x_{n}\right)$.

This expression was obtained by taking the spatial Fourier transform of the time-independent radiative transport equation over the transverse directions (i.e., $x$ - and $y$-dimensions). It provides the final weight of the $n$ 'th detected photon originating from a point source at $x_{o}=0$ with unity initial weight. Here, $d_{i, n}$ stands for the total photon path length in the $i$ 'th layer, and subscripts 1 and 2 refer to the top and bottom layers, respectively, of the two-layer tissue model. In addition to the effect of absorption (first exponential term), as in the case of a conventional continuous absorption-weighted MC simulation, the photon weight exhibits the effect of spatial modulation as a frequency-dependent phase accumulation (second exponential 
term), which is sensitive only to the net lateral displacement $\left(x_{n}\right)$ of the photon's exit location relative to the source. Equation (2) can then be used to compute $R_{d}$ as a function of $f_{x}$, where $N$ is the total number of photons simulated

$R_{d}\left(f_{x}\right)=\frac{1}{N} \sum_{n=1}^{N} W_{n}$.

Provided that $d_{1, n}, d_{2, n}$, and $x_{n}$ are stored, it is possible to compute $R_{d}$ for various spatial frequencies and $\mu_{a}$ values from a single MC simulation. This method of scaling for $\mu_{a}$ is more accurate than applying Beer's law to $R_{d} \cdot{ }^{28,30}$ A separate $\mathrm{MC}$ simulation must be run to obtain results for each $\mu_{s}^{\prime}$ value of interest.

In this work, we compare $R_{d}$ and optical property extractions from LUT inverse models based on both the conventional MC simulations scaled with the methods described in Martinelli et al., as well as MC simulations conducted with the methods described in Gardner et al. For convenience, we will refer to the MC methods used to generate LUTs as either the "Martinelli method" or "Gardner method." Similarly, the different LUTs used to produce $R_{d}$ and optical property extraction will be referred to as "Martinelli homogeneous," "Gardner homogeneous," and "Gardner two-layer."

\subsubsection{Monte Carlo simulation parameters for generating homogeneous and two-layer LUTS}

The Martinelli method was used to construct a homogeneous LUT, and the Gardner method was used to construct both a homogeneous LUT and a two-layer LUT. The geometries of the MC simulations used to produce these LUTs are shown in Figs. 1(a) and 1(b). Both models were constructed for the purpose of extracting optical properties from a subcutaneously implanted tumor on the mouse flank. For the homogeneous case, the tumor was modeled as a semi-infinite geometry, and the effects of the superficial skin layer were ignored. For the two-layer case, the top layer represents the skin layer, with fixed (i.e., known) $\mu_{a}, \mu_{s}^{\prime}$, and thickness (d), and the bottom layer represents a semi-infinite tumor layer. For all LUTs, the tumor optical properties are the free parameters of the inversion algorithm. We describe the properties of each layer in more detail below.

Skin layer: Optical properties of the upper (skin) layer of the MC simulations were adapted from Sabino et al., ${ }^{31}$ who recently reported $\mu_{a}$ and $\mu_{s}^{\prime}$ of skin from BALB/c male mice using a Kubelka-Munk model of photon transport and spectrophotometric measurements for the wavelength range of 400 to $1400 \mathrm{~nm}$. The authors used skin from the mouse dorsal region and shaved any excess hair before measurements. We utilized the average $\mu_{a}$ and $\mu_{s}^{\prime}$ values of the reported skin properties calculated over the four SFDI wavelengths $(659,691,731$, and $851 \mathrm{~nm})$ for the upper layer properties in our two-layer MC simulations: $\mu_{a}=0.096 \mathrm{~mm}^{-1}$ (SD: $0.0075 \mathrm{~mm}^{-1}$ ) and $\mu_{s}^{\prime}=0.78 \mathrm{~mm}^{-1}$ (SD: $0.12 \mathrm{~mm}^{-1}$ ).

The thickness of the skin layer was estimated using caliper measurements of eight excised tumor skin samples from C57BL/6N female mice. The average skin thickness, which included the epidermis, dermis, and hypodermis, was $312.5 \mu \mathrm{m}$. $\mathrm{H} \& \mathrm{E}$ staining of representative tumor skin cross sections was conducted to validate the skin thicknesses (agreement was within $3.1 \%$ for $n=2$ samples). The C57BL/6N mouse strain was used to estimate thickness since it is commonly used for mouse tumor imaging, ${ }^{32}$ and we plan to utilize this strain in future studies of mammary carcinoma. Our thickness measurements were similar to past reports of C57BL/6N skin thickness, where female mice were found to have a skin thickness of $371,{ }^{33}$ $300,{ }^{34}$ and $364 \mu \mathrm{m} .{ }^{31}$ We note that data collected from male severe combined immunodeficient (SCID) mice are used in Sec. 3.5 of this work as well as in our prior study. ${ }^{11}$ The thickness of male SCID mouse skin was determined by measuring 18 skin samples taken prior to treatment $(n=6)$, during treatment with either DC101 or cyclophosphamide (CPA) $(n=6)$, and after treatment $(n=6)$ using brightfield microscopy of frozen tissue sections. The average skin thickness was found to be $326.9 \mu \mathrm{m}$, which is within $5 \%$ of the thickness used in the two-layer model $(312.5 \mu \mathrm{m})$. The difference between the thickness of samples taken before and during or after treatment was within $11 \%$. These results suggest that the two-layer model is appropriate for subcutaneous tumor models in both female C57BL/6N and male SCID mice. Other mouse strains have somewhat similar skin thickness, including the commonly used BALB/c mouse strain (336 $\mu \mathrm{m}$ for female and $393 \mu \mathrm{m}$ for male) ${ }^{31}$ immunocompetent albino mice $(441 \mu \mathrm{m}),{ }^{35}$ immunocompromised athymic nude mice $(420 \mu \mathrm{m}),{ }^{34}$ and female SCID mice $(220 \mu \mathrm{m}){ }^{36}$

Tumor layer: In all three LUTs, the bottom (tumor) layer contains $400 \mu_{a}$ values, ranging from 0.0005 to $0.2 \mathrm{~mm}^{-1}$ $\left(\Delta \mu_{a}=0.0005 \mathrm{~mm}^{-1}\right)$ and $192 \mu_{s}^{\prime}$ values ranging from 0.1 to $3.58 \mathrm{~mm}^{-1}\left(\Delta \mu_{s}^{\prime}=0.018 \mathrm{~mm}^{-1}\right)$. A separate MC simulation was run for each $\mu_{s}^{\prime}$ value for both the homogeneous and two-layer Gardner LUTs. The tumor layer depth in all simulations was set to be at least 20 times the maximum $l^{*}\left[=1 /\left(\mu_{a}+\mu_{s}^{\prime}\right)\right]$ to mimic a semi-infinite tissue geometry, (a) Homogeneous medium

Spatially modulated illumination

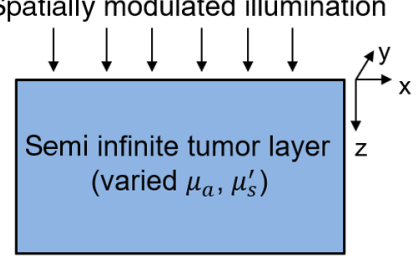

(b) Two-layer medium

Spatially modulated illumination

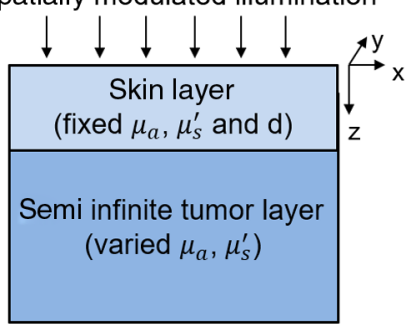

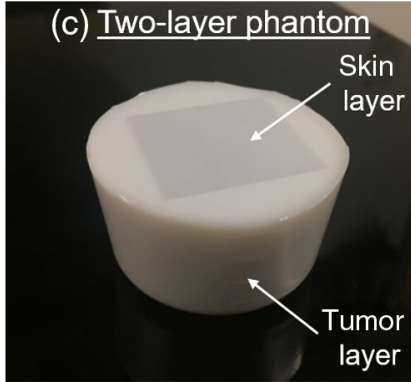

Fig. 1 (a) Schematic of tissue model for the homogeneous case, (b) schematic of the tissue model for the two-layer case, and (c) an example of a custom-made two-layer silicone phantom used to validate the accuracy of the resulting two-layer inverse algorithm. 
where $l^{*}$ was determined using the lowest $\mu_{a}$ value $\left(0.0005 \mathrm{~mm}^{-1}\right)$. Based on this criterion, a tumor layer thickness of $100 \mathrm{~mm}$ was used for all MC simulations except for the lowest $16 \mu_{s}^{\prime}$ values $\left(0.1\right.$ to $\left.0.37 \mathrm{~mm}^{-1}\right)$, for which the thickness was increased to $200 \mathrm{~mm}$.

\subsubsection{Monte Carlo simulations at Boston University shared computer cluster}

All MC simulations were conducted at the Boston University shared computer cluster located in Holyoke, Massachusetts. Previously developed command-line implementation of the Gardner method was used for the MC simulations (software developed by and implemented with the assistance of the Virtual Photonics Technology Initiative at the Beckman Laser Institute, University of California, Irvine, California). In all simulations and for both layers, the index of refraction and anisotropy factor ( $g$ ) was set to 1.4 (Ref. 37) and 0.9 (Ref. 38), respectively, except for simulations performed in Sec. 3.1, in which the index of refraction and $g$ for both layers were set to 1.33 and 0.71 , respectively, to match those used in Cuccia et al. ${ }^{12}$ We launched $1 \times 10^{7}$ photons for each simulation. ${ }^{12}$ A total of $192 \mathrm{MC}$ simulations were conducted to generate the Gardner LUTs, one for each $\mu_{s}^{\prime}$ value of the tumor layer. In these 192 simulations, $\mu_{a}$ was set to a value of $0.0005 \mathrm{~mm}^{-1}$ for the tumor layer. The 192 simulations were divided into 12 groups, each with 16 simulations, which were run in parallel. Each MC simulation required 2 CPUs, and runtime was dependent on optical properties and ranged from 2.5 to 4 days. The total photon path length in each layer and the photon exit position were stored for each simulation. The results from each simulation were postprocessed using Beer's law [as in Eq. (1)] to achieve results for all values of $\mu_{a}$. Postprocessing runtime was 2 to 3 days, depending on the specific optical properties.

\subsection{Two-Layer Tissue-Simulating Optical Phantoms}

A set of two-layer solid silicone phantoms was fabricated to optically simulate subcutaneous tumors in a mouse with a range of optical properties. These phantoms were used to test the accuracy of the Gardner LUT inversion algorithms. The phantoms consisted of a thin skin layer above a tumor layer. The top layer thickness and optical properties were fabricated to closely match the parameters described for MC simulations in Sec. 2.2.2. Four different two-layer phantoms were fabricated, all of which used the same skin layer. In all phantoms, silicone was used as the base solvent, nigrosin as the absorber, and titanium dioxide as the scatterer. The optical properties of the phantoms were adjusted by varying the amount of absorber and scatterer during fabrication as previously described. ${ }^{39}$

The thin upper layer phantom was made by adapting a previously described technique. ${ }^{40}$ First, an aluminum phantom mold was fabricated by machining a well that was $330 \mu \mathrm{m}$ in depth and $1.5^{\prime \prime} \times 1.5^{\prime \prime}$ in the lateral dimensions using a computer-controlled milling machine (SV-2414S-M, Sharp Industries). After the phantom ingredients were mixed together, the liquid mixture was poured into the aluminum mold. A microtome blade was used to draw and spread the mixture evenly across the well, and the edges of the blade remained in contact with the top surface of the mold at all times. The phantom was then left to cure, uncovered, overnight. During curing, the silicone layer was observed to shrink in the center of the well. Once cured, the thin silicone layer was removed from the mold and cut to the size of $1^{\prime \prime} \times 1^{\prime \prime}$ to remove the uneven and thicker edge. The thickness of the phantom was confirmed using caliper measurements by confining the thin layer between two microscope slide coverslips for stability and consistency. Because the top layer phantom was too thin for accurate optical property measurements with diffuse imaging techniques, a much larger, $2.5-\mathrm{cm}$ thick homogeneous phantom was made from the same batch of material and SFDI was used to extract the optical properties.

Similarly, for the bottom (tumor) layer, four homogeneous phantoms were fabricated in collaboration with Dr. Muldoon's group $^{41}$ and measured with SFDI. The thickness of each phantom was $2.5 \mathrm{~cm}$, and the $\mu_{a}$ and $\mu_{s}^{\prime}$ values of each phantom were targeted to span known mouse tumor optical properties. The skin layer and tumor layer phantoms were stacked to create the two-layer phantoms. First, the thin skin layer phantom was cleaned using an alcohol wipe. Then a small amount of ethanol was poured on a thick tumor layer phantom, and the thin layer was directly placed on top of the tumor layer, making sure that no visible air bubbles remained. The two-layer phantom was left under the chemical hood overnight to allow the ethanol to evaporate without leaving any air pockets between the layers. An example of one of the two-layer phantoms is shown in Fig. 1(c). The procedure was repeated 4 times to generate the four two-layer phantoms.

\subsection{Longitudinal Monitoring of a Mouse Tumor Xenograft During Cancer Treatment}

In our prior work, we conducted SFDI longitudinal monitoring of the PC3/2G7 prostate tumor xenograft model during treatment with anticancer agents. ${ }^{11,42} \mathrm{We}$ have reprocessed a portion ( $n=2$ mice) of this longitudinal data using the LUT inversion algorithms presented in this work in order to visualize the effect of using the multilayer model in a relevant physiologic system. Key details of the longitudinal study are presented here: SCID hairless outbred mice (SHO Mouse, Crl:SHOPrkdcscidHrhr), age 5 to 6 weeks old (21 to 23 grams), were purchased from Charles River Laboratories. When the average tumor volume reached $\sim 500 \mathrm{~mm}^{3}$, mice were treated with either the cytotoxic anticancer drug CPA (every 6 days for 3 cycles) or the antiangiogenic agent DC101 (every 3 days for 6 cycles), both given with intraperitoneal injections. During SFDI measurements, mice were anesthetized using isoflurane by inhalation. Mice were monitored longitudinally with SFDI for a total of 45 days. SFDI measurements were taken 5 times during 17 days of tumor growth, every day during 18 days of treatment, and every 2 days following the treatment period. Additional details including methods for tumor cell preparation, tumor cell inoculation, animal handling and care, SFDI measurement repeatability, angle and height corrections, etc., are described in Tabassum et al. ${ }^{25}$ All animal procedures and measurements were conducted under an institutionally approved protocol.

\section{Results}

\subsection{Comparison between MC Simulation Results and LUT Inversion Algorithms: Diffuse Reflectance}

We first compared the results of the three different MC simulations methods (i.e., Martinelli homogeneous, Gardner 


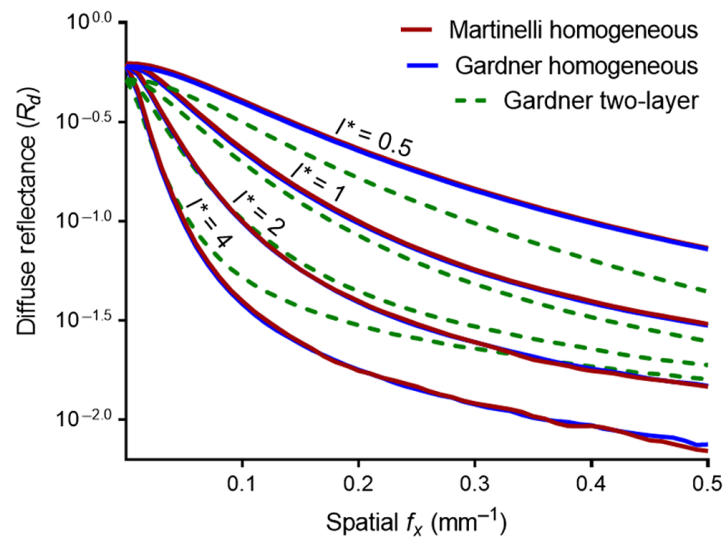

Fig. 2 Comparison of $M C$ simulation results from the Martinelli homogeneous, the Gardner homogeneous, and the Gardner twolayer methods. Diffuse reflectance $\left(R_{d}\right)$ is shown as a function of spatial frequency $\left(f_{X}\right)$ for varying values of $l^{*}$ at a constant ratio of $\mu_{s}^{\prime} / \mu_{a}=100$. The Martinelli homogeneous and Gardner homogeneous results are nearly identical, while the introduction of the top (skin) layer introduces significant shifts in $R_{d}$ that are dependent on $f_{x}$ and the $l^{*}$ of the bottom (tumor) layer. homogeneous, and Gardner two-layer) and then compared how the LUT inversion algorithms based on these simulations differently map $R_{d}$ values to optical property values.

Figure 2 shows an illustrative example of the differences and similarities in the MC simulation results. $R_{d}$ is shown as a function of $f_{x}$ for varying $l^{*}$ values at a constant ratio of $\mu_{s}^{\prime} / \mu_{a}=100$. The optical properties corresponding to each $l^{*}$ value are as follows: for $l^{*}=0.5 \mathrm{~mm}: \mu_{a}=0.0198 \mathrm{~mm}^{-1}, \mu_{s}^{\prime}=1.98 \mathrm{~mm}^{-1}$; $l^{*}=1 \mathrm{~mm}: \mu_{a}=0.0099 \mathrm{~mm}^{-1}, \quad \mu_{s}^{\prime}=0.99 \mathrm{~mm}^{-1} ; l^{*}=2 \mathrm{~mm}$ : $\mu_{a}=0.005 \mathrm{~mm}^{-1}, \quad \mu_{s}^{\prime}=0.5 \mathrm{~mm}^{-1} ;$ and $l^{*}=4 \mathrm{~mm}: \mu_{a}=$ $0.0025 \mathrm{~mm}^{-1}, \mu_{s}^{\prime}=0.25 \mathrm{~mm}^{-1}$. For the two-layer simulations, the skin layer properties were as previously described $\left(\mu_{a}=0.096 \mathrm{~mm}^{-1}, \mu_{s}^{\prime}=0.78 \mathrm{~mm}^{-1}, d=312.5 \mu \mathrm{m}\right)$. From Fig. 2, it is evident that there is very little difference in MC simulation results between the Martinelli homogeneous and the Gardner homogeneous methods. However, the incorporation of the skin layer introduces a significant alteration in these results, as expected. Note that the effect of the skin layer is to sometimes shift $R_{d}$ values higher than the homogeneous results and to sometimes shift them lower. This effect is dependent on spatial frequency as well as the specific optical properties of the tumor and skin layers. Because the Martinelli and Gardner homogeneous results are nearly identical, the remainder of the analysis will focus on the Gardner homogeneous and Gardner two-layer (a) Gardner homogeneous LUT (DC $R_{d}$ )

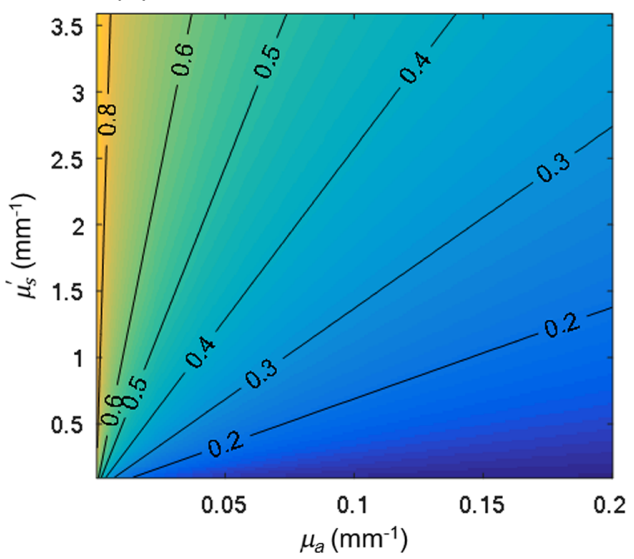

(c) Gardner homogeneous LUT (AC $R_{d}$ )

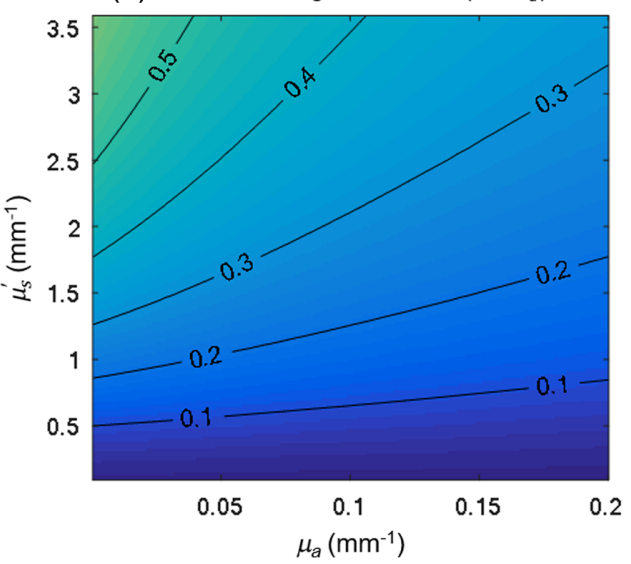

(b) Gardner two-layer LUT (DC $R_{d}$ )

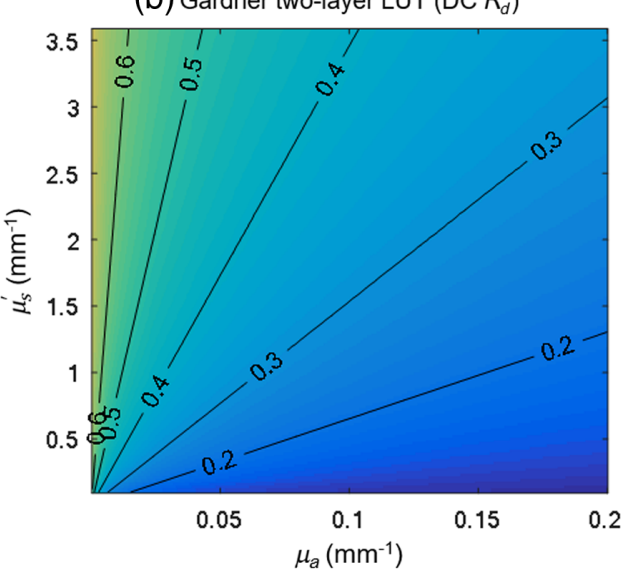

(d) Gardner two-layer LUT (AC $\left.R_{d}\right)$

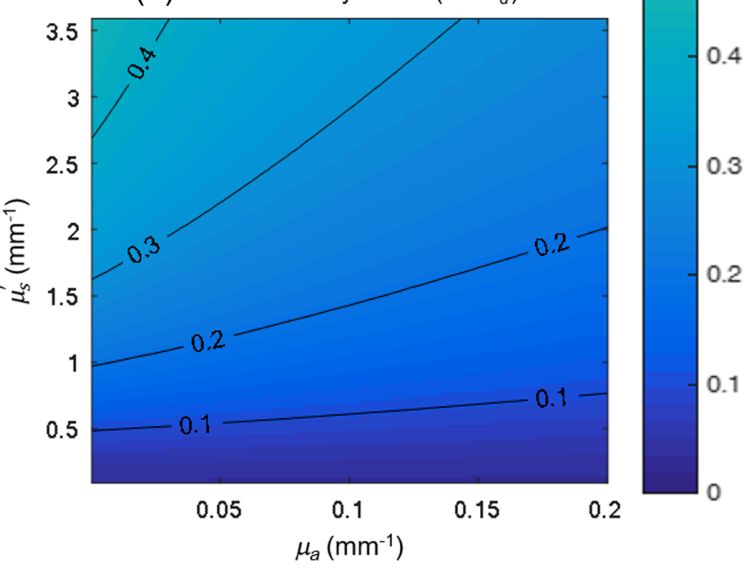

Fig. 3 Comparison of LUT inversion algorithms based on Gardner homogeneous and Gardner two-layer methods. $R_{d}$ values are shown both in the color dimension and as labeled isolines for the entire range of simulated $\mu_{a}$ and $\mu_{s}^{\prime}$ values. (a) and (b) Optical properties versus DC $R_{d}$ for the homogeneous and twolayer LUTs, respectively. (c) and (d) Optical properties versus AC $R_{d}$ for the homogeneous and two-layer LUTs, respectively. 
MC results and the LUT inversion algorithms based on these results.

Figure 3 shows differences between the Gardner homogeneous and Gardner two-layer LUT inversion algorithms for two spatial frequencies: $f_{x}=0 \mathrm{~mm}^{-1}\left(\mathrm{DC} R_{d}\right)$ and $0.1 \mathrm{~mm}^{-1}$ (AC $\left.R_{d}\right)$. Here, the $x$-axis displays $\mu_{a}$, the $y$-axis displays $\mu_{s}^{\prime}$, and the color axis displays $R_{d}$. Isolines of constant $R_{d}$ are displayed as an aid to visual comparisons between the subplots. As in Fig. 2, these plots visually show the substantial impact that the skin layer has on the mapping between diffuse reflectance and optical properties.

\subsection{Comparison between LUT Inversion Algorithms: Optical Property Extraction}

In practice, the LUT inversion algorithms accept experimentally measured $R_{d}$ values as inputs and provide optical property extractions as outputs. We investigated the extent to which the LUTs provide different optical property extractions for the same input measurements (i.e., $R_{d}$ values). To do this, we first choose $30 \mathrm{DC} R_{d}$ values evenly spaced between 0.0419 and 0.749 , and $30 \mathrm{AC} R_{d}$ values, evenly spaced between 0.0289 and 0.4514 . This range of $R_{d}$ values was chosen as they are present in both the homogeneous and two-layer LUTs. For all combinations of the chosen DC and $\mathrm{AC} R_{d}$ values, $\mu_{a}$ and $\mu_{s}^{\prime}$ were extracted using both LUTs, and the difference in $\mu_{a}$ and $\mu_{s}^{\prime}$ extractions was computed. Absolute differences in optical properties between the LUTs are shown in Figs. 4(a) and 4(c) for $\mu_{a}$ and $\mu_{s}^{\prime}$, respectively, and the percent differences (relative to the homogeneous LUT values) are shown in Figs. 4(b) and 4(d). For some DC and AC $R_{d}$ combinations, the differences in optical property extractions are substantial. For example, from Fig. 4(b), we see that percent differences for $\mu_{a}$ are larger $(\sim 60 \%$ to $80 \%)$ at higher DC $R_{d}$ values. In Fig. 4(d), larger differences in $\mu_{s}^{\prime}$ extractions occur at higher AC $R_{d}$ values. This analysis shows that the two LUTs differently map optical properties from $R_{d}$ inputs. In the following section, we demonstrate that the two-layer LUT improves the accuracy of tumor layer extractions.

\subsection{Two-Layer LUT Improves the Accuracy of Tumor Layer Optical Property Extractions Using SFDI}

Experimental measurements were conducted to determine if the Gardner two-layer LUT inversion algorithm improves the accuracy of tumor layer optical property extractions compared to the Gardner homogeneous LUT. This accuracy test utilized the four two-layer phantoms described in Sec. 2.3. Each of the two-layer phantoms used the same top (skin) layer. The measured thickness of the skin layer was $310 \mu \mathrm{m}$ at its center, which is within $0.8 \%$ of the skin layer thickness defined in the MC simulations used to generate the Gardner two-layer LUT. Absorption of the skin layer was $0.0936 \mathrm{~mm}^{-1}$ at $659 \mathrm{~nm}$, which is within $2.52 \%$ of the MC absorption parameter, and the $\mu_{s}^{\prime}$ value was $0.780 \mathrm{~mm}^{-1}$ at $659 \mathrm{~nm}$, which is within $0.063 \%$ of the MC value. For the tumor layer, four different pairs of optical properties were utilized, spanning a range of optical properties
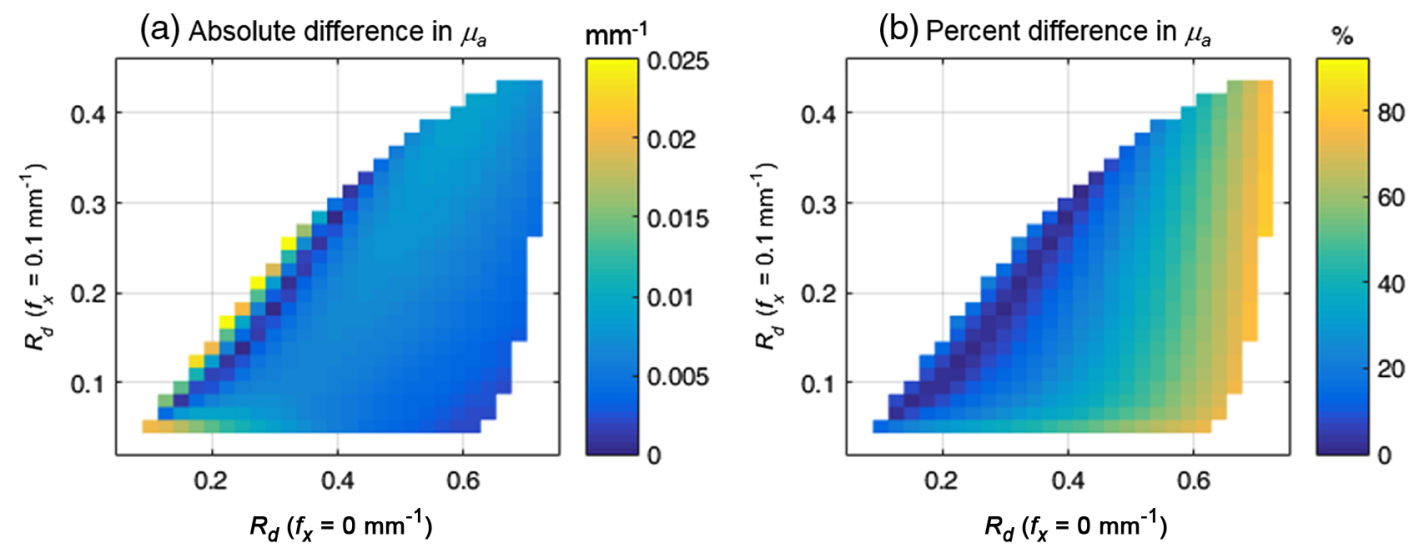

(c) Absolute difference in $\mu_{s}^{\prime}$

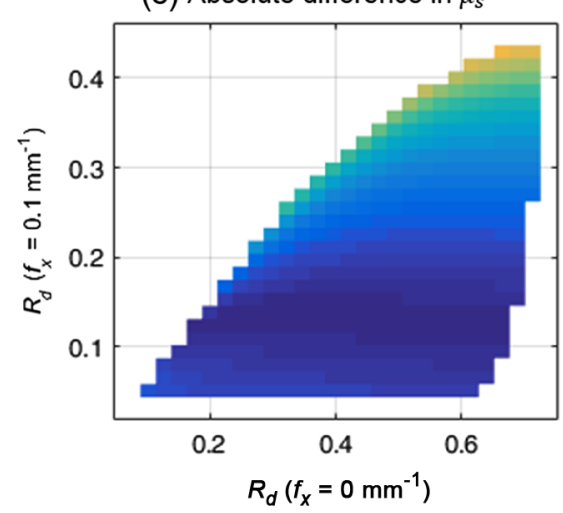

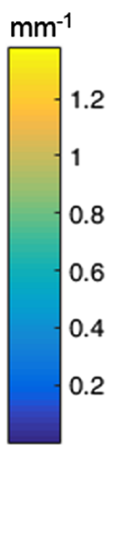

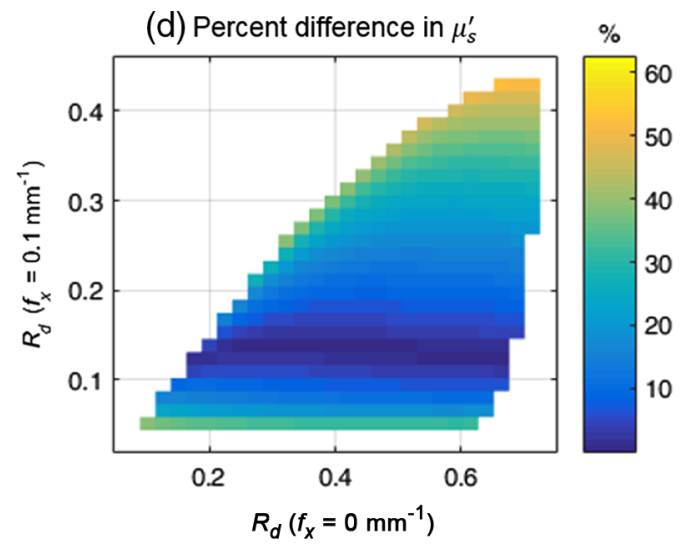

Fig. 4 The impact on optical property extractions of the Gardner two-layer LUT inversion algorithm shown as absolute and \% differences compared to the Gardner homogeneous case. (a) Absolute and (b) percent differences in $\mu_{a}$ extractions. (c) Absolute and (d) percent difference in $\mu_{s}^{\prime}$ extractions. 
(a)

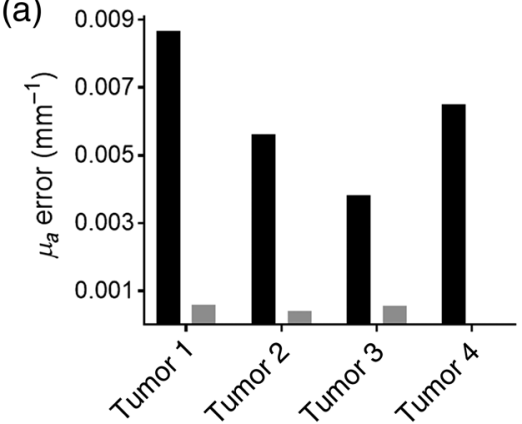

(c)

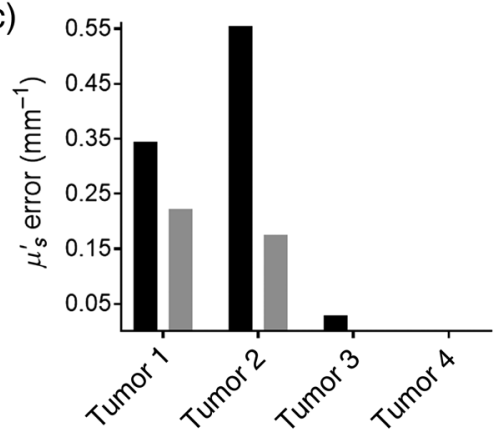

(b)

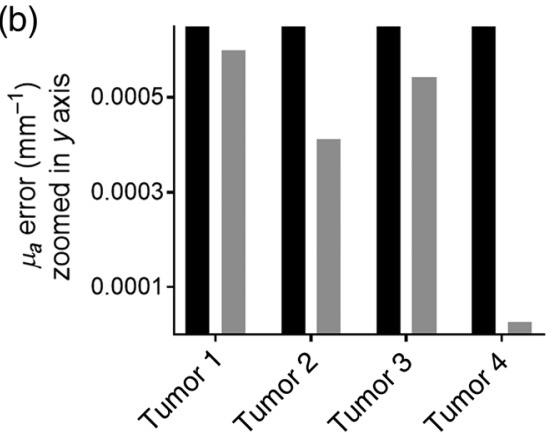

(d)

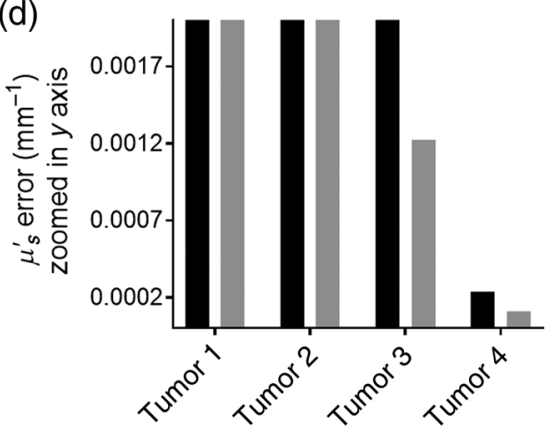

Gardner

homogeneous

Gardner

two-layer

Tumor 1:

High $\mu_{a}=0.0244 \mathrm{~mm}^{-1}$

High $\mu_{s}^{\prime}=2.054 \mathrm{~mm}^{-1}$

Tumor 2:

Low $\mu_{a}=0.002 \mathrm{~mm}^{-1}$

High $\mu_{s}^{\prime}=2.314 \mathrm{~mm}^{-1}$

Tumor 3:

Low $\mu_{2}=0.0039 \mathrm{~mm}^{-1}$

Low $\mu_{s}^{\prime}=0.714 \mathrm{~mm}^{-1}$

Tumor 4:

High $\mu_{a}=0.0301 \mathrm{~mm}^{-1}$

Low $\mu_{s}^{\prime}=0.676 \mathrm{~mm}^{-1}$

Fig. 5 Comparisons in bottom (tumor) layer optical property extraction errors for the Gardner homogeneous and Gardner two-layer LUT inversion algorithms. Diffuse reflectance measurements of four two-layer tissue-simulating optical phantoms were made with SFDI, and both inversion models were used to extract the bottom (tumor) layer optical properties (labeled as tumors 1 to 4). (a) The absolute extraction error compared with the known tumor layer $\mu_{a}$. (b) The same data but with a zoomed-in $y$-axis so that small extraction errors can be visualized. (c) Absolute errors in tumor layer $\mu_{s}^{\prime}$ extractions and (d) the same data with a zoomed-in $y$-axis. Optical properties were measured at $659 \mathrm{~nm}$.

observed in our prior work, in which we monitored PC3/2G7 mouse xenografts over 45 days using SFDI. ${ }^{11}$ These pairs are labeled as tumor 1 through tumor 4 in Fig. 5. The optical property pairs, reported at $659 \mathrm{~nm}$, are as follows: for tumor 1: $\mu_{a}=0.0244 \mathrm{~mm}^{-1}$ and $\mu_{s}^{\prime}=2.054 \mathrm{~mm}^{-1}$; tumor 2 : $\mu_{a}=0.002 \mathrm{~mm}^{-1}$ and $\mu_{s}^{\prime}=2.314 \mathrm{~mm}^{-1}$; tumor 3: $\mu_{a}=$ $0.0039 \mathrm{~mm}^{-1}$ and $\mu_{s}^{\prime}=0.714 \mathrm{~mm}^{-1}$; and tumor $4: \mu_{a}=$ $0.0301 \mathrm{~mm}^{-1}$ and $\mu_{s}^{\prime}=0.676 \mathrm{~mm}^{-1}$.

Each two-layer phantom was measured with SFDI, and the bottom (tumor) layer optical properties were extracted using both the Gardner homogeneous and Gardner two-layer LUTs. Since these phantoms have flat surfaces, no corrections for height or angle were implemented. The absolute differences between the measured and true $\mu_{a}$ for the tumor layer are shown in Figs. 5(a) and 5(b). The absolute differences between the measured and true $\mu_{s}^{\prime}$ for the tumor layer are shown in Figs. 5(c) and 5(d). In both cases, Figs. 5(b) and 5(d) recapitulates the data in Figs. 5(a) and 5(c) but with a zoomed-in $y$-axis to allow visualization of the small error values obtained for some phantoms. Table 1 shows the percent error in tumor layer optical property extractions for both the homogeneous and two-layer LUTs. In the worst-case, the $\mu_{a}$ and $\mu_{s}^{\prime}$ extraction errors were $20.33 \%$ and $10.87 \%$ for the two-layer LUT.

In all cases, the error in tumor layer optical property extraction is substantially lower for the two-layer LUT versus the homogeneous LUT. This effect is not as pronounced in $\mu_{s}^{\prime}$ for tumors 3 and 4 , as $\mu_{s}^{\prime}$ values in these tumors are very similar to that of the skin layer $\left(\mu_{s}^{\prime}=0.78 \mathrm{~mm}^{-1}\right)$. Note that the decrease in error by the two-layer LUT is between 7 and 256 times for $\mu_{a}$ and between 2 and 24 times for $\mu_{s}^{\prime}$. Taken together,
Table 1 Accuracy of optical property extractions in four two-layer tissue-simulating optical phantoms. Each two-layer phantom is designated as tumors 1 to 4 .

\begin{tabular}{lccccc} 
& \multicolumn{4}{c}{$\%$ error } \\
\cline { 2 - 3 } \cline { 5 - 6 } \cline { 5 - 6 } & Homogeneous & Two-layer & & Homogeneous & Two-layer \\
\cline { 2 - 3 } Tumor 1 & 35.45 & 2.46 & & 16.77 & 10.87 \\
Tumor 2 & 277.03 & 20.33 & & 23.98 & 7.62 \\
Tumor 3 & 97.72 & 13.88 & & 4.05 & 0.17 \\
Tumor 4 & 21.65 & 0.08 & & 0.03 & 0.02 \\
\hline
\end{tabular}

these results confirm that the two-layer LUT provides a better estimate of the true tumor layer optical properties than the homogeneous LUT.

\subsection{Sensitivity Analysis of the Two-Layer LUT}

We conducted a sensitivity analysis to characterize how mismatches in the skin layer optical properties and thickness affect the results of the Gardner two-layer LUT. Stated another way, the sensitivity analysis provides an indication of how well one must know the true skin layer properties in order to obtain accurate tumor layer optical property extractions. 
Additional MC simulations were conducted of a two-layer medium, in which the properties of the skin layer $\left(\mu_{a}, \mu_{s}^{\prime}\right.$, and $d$ ) were varied to introduce a mismatch to those used to generate the Gardner two-layer LUT. The skin layer $\mu_{a}$ value was varied by up to $\pm 40 \%$ of the original Gardner two-layer value, $\mu_{s}^{\prime}$ was varied by up to $\pm 40 \%$, and $d$ was varied from $-40 \%$ to $+80 \%$. Unlike optical properties, $d$ was varied by up to $+80 \%$ in order to simulate large skin thickness values, such as $d=530 \mu \mathrm{m}$, reported previously in athymic nude mouse. ${ }^{34}$ For this analysis, four tumor layer optical property (a)

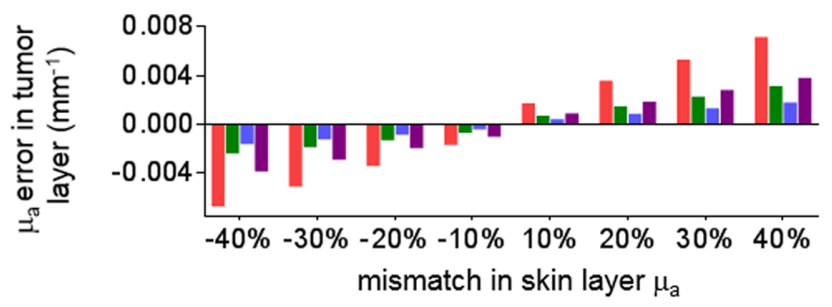

(b)
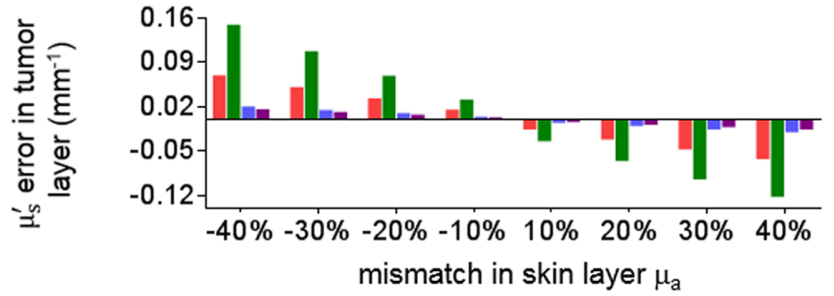

(c)

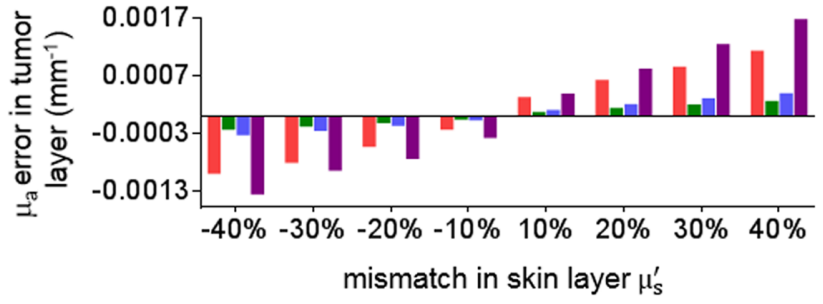

(d)
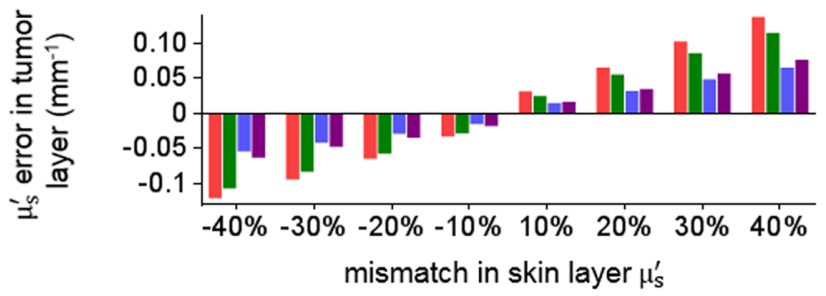

(e)

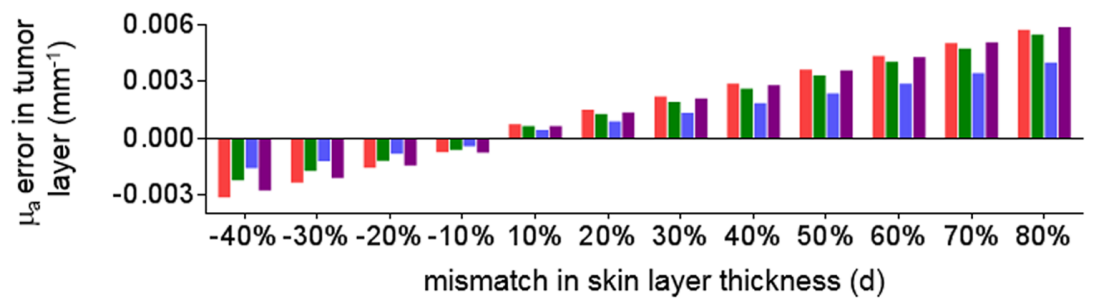

(f)

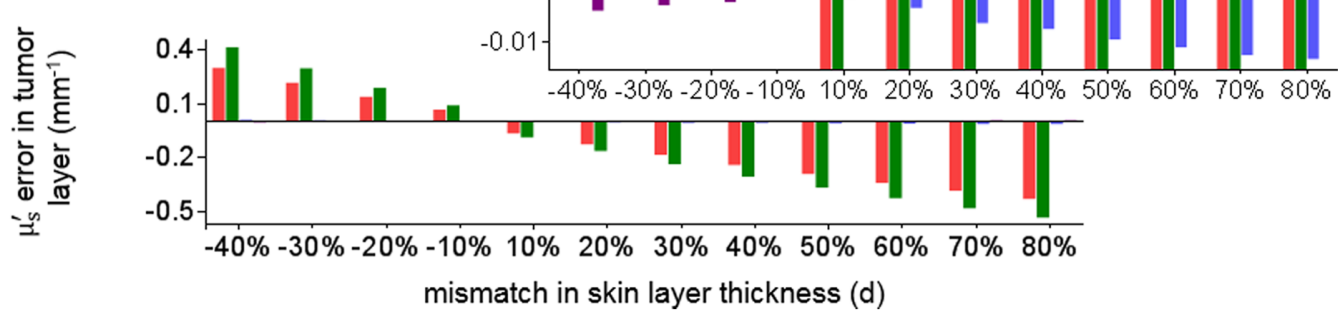

Fig. 6 Results from a sensitivity analysis for the two-layer Gardner LUT inversion algorithm. Errors in tumor layer $\mu_{\mathrm{a}}$ and $\mu_{s}^{\prime}$ extractions are shown for various skin layer property mismatches (a)-(f). In (f), the inset image has a zoomed-in $y$-axis. Optical properties were measured at $659 \mathrm{~nm}$. 
pairs were chosen to span a physiologically relevant range, and extraction errors are reported for each pair. The tumor layer optical property pairs at $659 \mathrm{~nm}$ are listed here: For tumor 1: $\mu_{a}=0.031 \mathrm{~mm}^{-1}$ and $\mu_{s}^{\prime}=2.025 \mathrm{~mm}^{-1}$; tumor 2: $\mu_{a}=0.004 \mathrm{~mm}^{-1}$ and $\mu_{s}^{\prime}=2.2 \mathrm{~mm}^{-1}$; tumor 3 : $\mu_{a}=0.005 \mathrm{~mm}^{-1}$ and $\mu_{s}^{\prime}=0.676 \mathrm{~mm}^{-1}$; and tumor $4: \mu_{a}=$ $0.033 \mathrm{~mm}^{-1}$ and $\mu_{s}^{\prime}=0.645 \mathrm{~mm}^{-1}$.

In order to compute extraction errors induced by the mismatched skin layer, the $R_{d}$ values at the DC and AC spatial frequencies produced from each of the new MC simulations (which each had mismatched skin layer properties) were fed to the original Gardner two-layer LUT and resulting optical property extractions were recorded. The error was then computed, defined as the difference between the known tumor layer optical properties and the recorded optical property extractions. These errors are shown in Figs. 6(a) and 6(f). As expected, the errors in $\mu_{a}$ and $\mu_{s}^{\prime}$ extractions increase as the mismatch in skin layer properties increases. For example, as described in Sec. 2.2.2, Dodig et al. $^{36}$ reported that female SCID mice had a skin thickness of $\sim 220 \mu \mathrm{m}$. This is an approximately $-30 \%$ mismatch with the skin thickness used for our model (312.5 $\mu \mathrm{m})$. As shown in Figs. 6(e) and 6(f), assuming the skin optical properties match, this $-30 \%$ mismatch in skin layer thickness may induce an error in $\mu_{a}$ of as much as
$-0.0023 \mathrm{~mm}^{-1}$ and a $\mu_{s}^{\prime}$ error of as much as $0.29 \mathrm{~mm}^{-1}$ (these are the worst-case errors observed from this analysis, substantially smaller errors were observed for some tumor optical property combinations).

Additional MC simulations were conducted, in which the properties of the skin layer $\left(\mu_{a}, \mu_{s}^{\prime}\right.$, and $\left.d\right)$ were varied simultaneously to explore the effect of combining these mismatches. The maximum errors observed when all three parameters were mismatched in the negative direction (i.e., all three parameters decreased by $40 \%$ ) was $-0.0078 \mathrm{~mm}^{-1}$ for $\mu_{a}$ and $0.44 \mathrm{~mm}^{-1}$ for $\mu_{s}^{\prime}$. The maximum errors observed when all three parameters were mismatched in the positive direction (i.e., $\mu_{a}$ and $\mu_{s}^{\prime}$ increased by $40 \%, d$ increased by $80 \%$ ) was $0.021 \mathrm{~mm}^{-1}$ for $\mu_{a}$ and $-0.51 \mathrm{~mm}^{-1}$ for $\mu_{s}^{\prime}$. Whether errors of this magnitude are tolerable depending on the specific application and biological questions posed.

\subsection{Gardner Two-Layer LUT Reveals Larger Therapy-Induced Optical Scattering Dynamics and a More Hypoxic Tumor Environment During Longitudinal Monitoring of Tumor Xenografts}

The homogeneous and two-layer LUT inversion algorithms were used to reanalyze a prior data set, in which SFDI was (a) $\mu_{\mathrm{s}}^{\prime}$ in a DC101-treated prostate tumor

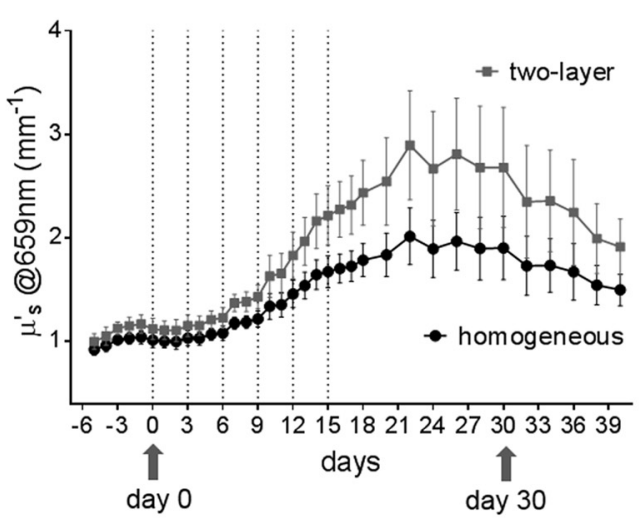

(c) $\mathrm{StO}_{2}$ in a Metronomic CPA-treated prostate tumor

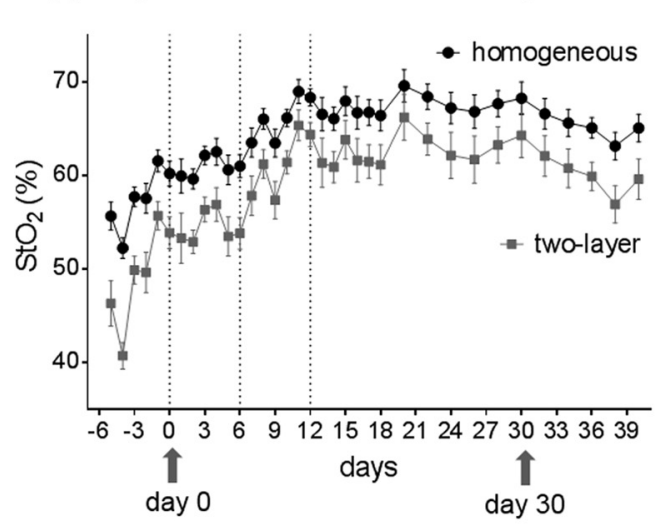

(b) $\mu_{\mathrm{s}}^{\prime}$
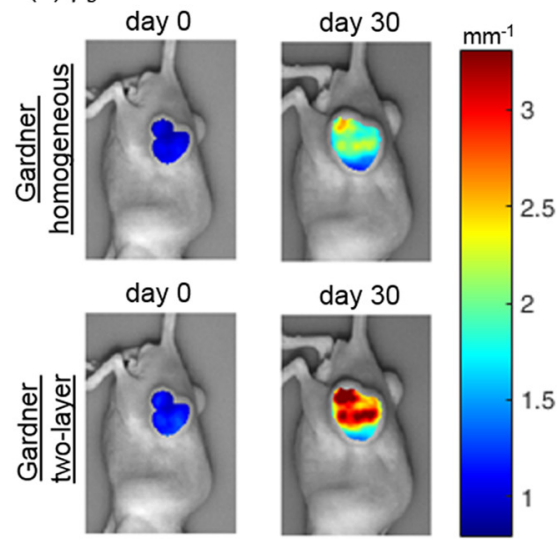

(d) $\mathrm{StO}_{2}$
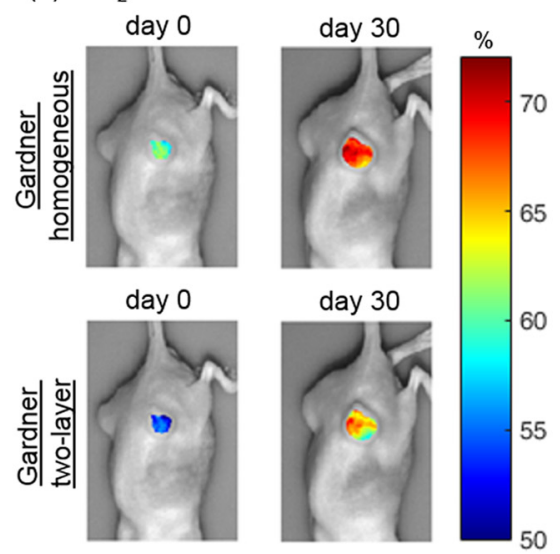

Fig. 7 An example of the Gardner homogeneous and the Gardner two-layer LUT inversion algorithms applied to SFDI data collected during a longitudinal treatment monitoring study of PC3/2G7 prostate tumor xenografts. (a) $\mu_{s}^{\prime}$ extractions from both LUTs are shown during and after DC101 treatment. (b) $\mu_{s}^{\prime}$ colormaps overlaid on $\mathrm{DC} 101$-treated planar mouse images at day 0 and day 30 . (c) $\mathrm{StO}_{2}$ values determined using four wavelength optical property extractions from both LUTs for a CPA-treated tumor. (d) $\mathrm{StO}_{2}$ colormaps overlaid on CPA-treated planar mouse images at day 0 and day 30 . 
used to monitor mice longitudinally during the course of anticancer therapy. ${ }^{11}$ The details of the data acquisition and analysis, including the methods for region of interest (ROI) selection, are described in detail in Tabassum et al. ${ }^{11}$ Briefly, each SFDI measurement was repeated thrice and averaged to minimize breathing artifacts. The demodulated images were corrected for height and angle. $^{24}$ Two-by-two binning of the CCD was applied to improve the SNR. Mice were monitored longitudinally for a total of 45 days. $R_{d}$ data acquired with SFDI were analyzed with both the Gardner homogeneous and Gardner two-layer LUT inversion algorithms for comparison.

Figure 7(a) shows changes in tumor $\mu_{s}^{\prime}$ at $659 \mathrm{~nm}$ from a DC101-treated tumor over the course of 45 days. Injection dates are indicated by vertical dashed lines. The mean and standard deviation of $\mu_{s}^{\prime}$ values extracted over a manually chosen ROI are shown. The two-layer LUT reveals higher $\mu_{s}^{\prime}$ values throughout the study compared to the homogeneous LUT, and the changes in $\mu_{s}^{\prime}$ over time are also larger. Figure 7(b) shows tumor $\mu_{s}^{\prime}$ colormaps overlaid on a planar mouse image at day 0 and day 30 for both LUTs. The increase in $\mu_{s}^{\prime}$ is apparent throughout the tumor region at these time points.

Figure $7(\mathrm{c})$ shows changes in tumor $\mathrm{StO}_{2}$ from a CPA-treated tumor on a metronomic schedule, followed by a rebound period. Mean and standard deviation of $\mathrm{StO}_{2}$ values extracted over a manually chosen ROI are shown. The two-layer LUT reveals lower $\mathrm{StO}_{2}$ values over the entire study period compared to the homogeneous LUT; the values decrease by roughly the same extent throughout the study. Figure 7(d) shows tumor $\mathrm{StO}_{2}$ colormaps overlaid on planar mouse images at day 0 and day 30 for both LUTs. A decrease in $\mathrm{StO}_{2}$ is apparent throughout the tumor region at these time points.

\section{Discussion and Conclusions}

In this work, a two-layer LUT inversion algorithm was introduced to more accurately account for the tumor and skin layered physiology in a small animal oncology model when imaging with SFDI. The LUT was constructed using MC simulation results conducted natively in the spatial frequency domain with the recently developed method by Gardner et al., which avoids the discretization errors associated with Fourier or Hankel transforming conventional spatially resolved MC results. ${ }^{29}$ The two-layer tissue LUT was superior in its ability to extract both $\mu_{a}$ and $\mu_{s}^{\prime}$ from the tumor layer, decreasing errors by as much as a factor of 256 for $\mu_{a}$ compared to a homogeneous LUT. The magnitude of the improvement was highly dependent on both the optical properties of the tumor layer and spatial frequencies considered. When applied to a longitudinal data set, the two-layer LUT revealed larger antitumor therapy-induced changes in tumors and a more hypoxic tumor environment.

One important characteristic of the two-layer model developed here is that the top (skin) layer optical properties and thickness $\left(\mu_{a}, \mu_{s}^{\prime}\right.$, and $\left.d\right)$ were fixed rather than free parameters. Estimates for these parameters were based on a prior report of male BALB/c albino mice skin optical properties and our own mouse skin thickness measurements. ${ }^{31}$ Unfortunately, there are limited reports of mouse skin optical properties in the literature, and skin thickness reports vary by mouse strain and gender. ${ }^{31,34}$ A sensitivity analysis was conducted to evaluate the impact of imperfect top layer assumptions on the extraction of bottom (tumor) layer optical properties. This analysis is useful for understanding the impact of using this two-layer LUT for mouse strains and genders with different optical properties or thicknesses, or for measurements in different wavelength regions. Care should be taken when this model is applied to other mouse strains and genders, as each application will have a different threshold for acceptable error.

The utility of the two-layer LUT was demonstrated by reanalyzing a prior longitudinal data set with the new model and comparing the results to a homogeneous LUT. The two-layer LUT revealed substantial differences compared to the homogeneous LUT during treatment with two anticancer therapies: DC101, a targeted antiangiogenic, and CPA, a cytotoxic agent. For example, larger increases in $\mu_{s}^{\prime}$ (up to $2.75 \times$ ) were observed in the DC101-treated tumor when analyzed with the two-layer LUT, and the optical contrast between pre- and posttreatment time points was enhanced significantly. In the CPA-treated tumor, the two-layer LUT revealed lower tumor $\mathrm{StO}_{2}$ throughout this study, including before baseline, during treatment, and during a rebound period. In both of these scenarios, the differences between the two-layer and homogeneous results are presumably due to the fact that the two-layer LUT is better able to isolate the tumor layer optical properties, whereas the homogeneous LUT convolves the changes in the tumor layer with the skin layer. It is of note that in some cases the twolayer LUT provides better agreement with other reported tumor values compared with the homogeneous LUT. For example, the baseline $\mathrm{StO}_{2}$ in the CPA-treated tumor was $60.2 \%$ with the homogeneous LUT and $53.9 \%$ with the two-layer LUT. In this case, the two-layer LUT $\mathrm{StO}_{2}$ value better matches the $\mathrm{StO}_{2}$ values of $40 \%$ to $55 \%$ reported for K1735 malignant mouse melanoma subcutaneous tumors measured using diffuse reflectance spectroscopy. ${ }^{43}$

There have been other reports of multilayer inversion models for SFDI, but they have largely focused on clinical applications in human skin, in which layer thicknesses, chromophores, and optical properties may be substantially different than those for mice. For example, Weber et al. ${ }^{17}$ developed an analytic twolayer model based on photon diffusion theory in the spatial frequency domain. The model had five fit parameters (i.e., top and bottom layers $\mu_{a}, \mu_{s}^{\prime}$, and top layer thickness), and provided bottom layer $\mu_{a}$ extractions with an accuracy of $25 \%$ when the top layer thickness was constrained to within $25 \%$ of the true value. This model was tested for top layer thicknesses of 2 to $4 \mathrm{~mm}$, which are substantially larger than the $312.5 \mu \mathrm{m}$ top layer thickness used in this work. Saager et al. ${ }^{20,21}$ utilized Hankel-transformed conventional (i.e., spatially resolved) MC simulations to develop a two-layer model of human epidermis (containing melanin) and dermis (containing melanin and hemoglobin). This model also provides estimates for top layer thickness constrained within a range of 80 to $300 \mu \mathrm{m}$. Yudovsky et al. ${ }^{18,19}$ also utilized Hankel-transformed conventional MC to create a two-layer model of human skin, with special attention to skin pigmentation and epidermal thickness. They used the MC results to train an artificial neural network in order to develop their inversion algorithm. The work presented here differs from these prior works in that the model parameters were specific to small animal tumor models, and the MC simulations used to generate the LUT-based inversion model were conducted natively in the spatial frequency domain.

While our work has demonstrated that a two-layer LUT model improves the optical property extraction accuracy of the bottom (tumor) layer for a mouse tumor model, there are some limitations that the reader should bear in mind. For 
example, it is currently unknown to what extent skin optical properties or thickness changes during treatment in different mouse tumor models, which could affect the ability to accurately extract tumor optical properties. Additionally, as previously discussed, this model assumed a fixed set of upper layer optical properties and thickness. This limits the applicability of the two-layer LUT to substantially different mouse models and different wavelength ranges. It is of note that the general methodology described here can be utilized to construct multilayer LUTs for other SFDI applications, including for different mouse models or clinical applications with different layer thicknesses and optical property ranges, but this requires additional MC simulations and data postprocessing. An additional limitation is that the two-layer model only accounted for skin as a single layer, whereas a more complex model might include separate epidermis, dermis, and hypodermis layers in addition to the tumor layer. It is conceivable that a more complex layered model would improve optical property extraction accuracy, but there are challenges associated with isolating and measuring the optical properties of each of these layers for use as fixed model parameters. It is possible to allow upper layer thickness and optical properties to be free parameters in the inverse model, but this substantially increases the solution space and may reduce the ability to accurately extract bottom-layer optical properties. ${ }^{17}$ It can also lead to underdetermined problems. ${ }^{18}$ Finally, we reported results only for spatial frequencies of DC and $0.1 \mathrm{~mm}^{-1}$. While the MC results can be postprocessed for arbitrary spatial frequencies, the choice to use this frequency pair was made based on our recent work utilizing CramérRao lower bounds to determine optical property uncertainty estimates for SFDI. ${ }^{26}$ This analysis revealed DC and $0.1 \mathrm{~mm}^{-1}$ as excellent choices to reduce optical property extraction uncertainty for similar optical property ranges.

In conclusion, the two-layer LUT model presented was shown to substantially improve the ability of SFDI to extract bottom (tumor) layer optical properties, and this revealed larger treatment changes in tumor optical properties and a more hypoxic tumor environment in a mouse tumor model. Since therapy-induced optical changes may be subtle for some drugs and tumor models, the ability of the two-layer LUT to provide more accurate and enhanced pre- and posttreatment tumor contrasts may substantially increase the utility of SFDI as a preclinical imaging tool for monitoring cancer treatments. In the future, the use of SFDI to accurately monitor therapies in small animal tumor models may provide an efficient platform to test new drugs and combination regimens prior to clinical translation. This could then inform the use of clinical DOI technologies and help establish these techniques as important feedback methods during cancer treatment.

\section{Disclosures}

The authors have no relevant financial interests in this paper and no potential conflicts of interest to disclose.

\section{Acknowledgments}

The authors gratefully acknowledge funding from the American Cancer Society (Grant No. RSG14-014-01-CCE), the U.S. Department of Defense (Grant No. W81XWH-15-1-0070), and the Boston University Cross-Disciplinary Training Program in Nanotechnology for Cancer (XTNC). The authors wish to thank Carole Hayakawa, Vasan Venugopalan, Amaan Mazhar, and David Cuccia for their technical assistance and helpful discussions.

\section{References}

1. A. M. Gonzalez-Angulo, F. Morales-Vasquez, and G. N. Hortobagyi, "Overview of resistance to systemic therapy in patients with breast cancer," Adv. Exp. Med. Biol. 608, 1-22 (2007).

2. Y. Minami and M. Kudo, "Imaging modalities for assessment of treatment response to nonsurgical hepatocellular carcinoma therapy: contrast-enhanced US, CT, and MRI," Liver Cancer 4(2), 106-114 (2015).

3. N. Cho et al., "Early prediction of response to neoadjuvant chemotherapy in breast cancer patients: comparison of single-voxel (1)H-magnetic resonance spectroscopy and (18)F-fluorodeoxyglucose positron emission tomography," Eur. Radiol. 26(7), 2279-2290 (2015).

4. I. J. Chou et al., "Subjective discomfort in children receiving 3 T MRI and experienced adults' perspective on children's tolerability of $7 \mathrm{~T}$ : a cross-sectional questionnaire survey," BMJ Open 4(10), e006094 (2014).

5. T. D. O'Sullivan et al., "Diffuse optical imaging using spatially and temporally modulated light," J. Biomed. Opt. 17(7), 071311 (2012).

6. B. E. Schaafsma et al., "Optical mammography using diffuse optical spectroscopy for monitoring tumor response to neoadjuvant chemotherapy in women with locally advanced breast cancer," Clin. Cancer Res. 21(3), 577-584 (2015).

7. S. Jiang et al., "Predicting breast tumor response to neoadjuvant chemotherapy with diffuse optical spectroscopic tomography prior to treatment," Clin. Cancer Res. 20(23), 6006-6015 (2014).

8. D. Roblyer et al., "Optical imaging of breast cancer oxyhemoglobin flare correlates with neoadjuvant chemotherapy response one day after starting treatment," Proc. Natl. Acad. Sci. U. S. A. 108 (35), 14626-14631 (2011).

9. M. G. Pakalniskis et al., "Tumor angiogenesis change estimated by using diffuse optical spectroscopic tomography: demonstrated correlation in women undergoing neoadjuvant chemotherapy for invasive breast cancer?" Radiology 259(2), 365-374 (2011).

10. S. H. Chung et al., "Macroscopic optical physiological parameters correlate with microscopic proliferation and vessel area breast cancer signatures," Breast Cancer Res. 17(1), 72 (2015).

11. S. Tabassum et al., "Feasibility of spatial frequency domain imaging (SFDI) for optically characterizing a preclinical oncology model," Biomed. Opt. Express 7(10), 4154-4170 (2016).

12. D. J. Cuccia et al., "Quantitation and mapping of tissue optical properties using modulated imaging," J. Biomed. Opt. 14(2), 024012 (2009).

13. A. Ponticorvo et al., "Quantitative assessment of partial vascular occlusions in a swine pedicle flap model using spatial frequency domain imaging," Biomed. Opt. Express 4(2), 298-306 (2013).

14. U. Sunar et al., "Quantification of PpIX concentration in basal cell carcinoma and squamous cell carcinoma models using spatial frequency domain imaging," Biomed. Opt. Express 4(4), 531-537 (2013).

15. D. J. Rohrbach et al., "Preoperative mapping of nonmelanoma skin cancer using spatial frequency domain and ultrasound imaging," Acad. Radiol. 21(2), 263-270 (2014).

16. J. Q. Nguyen et al., "Spatial frequency domain imaging of burn wounds in a preclinical model of graded burn severity," J. Biomed. Opt. 18(6), 066010 (2013).

17. J. R. Weber et al., "Noncontact imaging of absorption and scattering in layered tissue using spatially modulated structured light," J. Appl. Phys. 105(10), 102028 (2009).

18. D. Yudovsky, J. Q. M. Nguyen, and A. J. Durkin, "In vivo spatial frequency domain spectroscopy of two layer media," J. Biomed. Opt. 17(10), 107006 (2012).

19. D. Yudovsky and A. J. Durkin, "Spatial frequency domain spectroscopy of two layer media," J. Biomed. Opt. 16(10), 107005 (2011).

20. R. B. Saager et al., "Method for depth-resolved quantitation of optical properties in layered media using spatially modulated quantitative spectroscopy," J. Biomed. Opt. 16(7), 077002 (2011).

21. R. B. Saager et al., "In vivo isolation of the effects of melanin from underlying hemodynamics across skin types using spatial frequency domain spectroscopy," J. Biomed. Opt. 21(5), 057001 (2016). 
22. S. D. Konecky et al., "Spatial frequency domain tomography of protoporphyrin IX fluorescence in preclinical glioma models," J. Biomed. Opt. 17(5), 056008 (2012).

23. S. D. Konecky et al., "Quantitative optical tomography of sub-surface heterogeneities using spatially modulated structured light," Opt. Express 17(17), 14780-14790 (2009).

24. Y. Zhao et al., "Angle correction for small animal tumor imaging with spatial frequency domain imaging (SFDI)," Biomed. Opt. Express 7(6), 2373-2384 (2016)

25. S. Tabassum, R. Istfan, and D. Roblyer, "Longitudinal monitoring of therapy response in a preclinical model using spatial frequency domain imaging," in Biomedical Optics, OSA Technical Digest, p. JM3A.49 (2016).

26. V. Pera et al., "Optical property uncertainty estimates for spatial frequency domain imaging," Biomed. Opt. Express 9(2), 661-678 (2018).

27. Y. Zhao and D. Roblyer, "Spatial mapping of fluorophore quantum yield in diffusive media," J. Biomed. Opt. 20(8), 086013 (2015).

28. M. Martinelli et al., "Analysis of single Monte Carlo methods for prediction of reflectance from turbid media," Opt. Express 19(20), 19627-19642 (2011).

29. A. R. Gardner and V. Venugopalan, "Accurate and efficient Monte Carlo solutions to the radiative transport equation in the spatial frequency domain," Opt. Lett. 36(7), 2269-2271 (2011).

30. C. K. Hayakawa et al., "Perturbation Monte Carlo methods to solve inverse photon migration problems in heterogeneous tissues," Opt. Lett. 26(17), 1335-1337 (2001).

31. C. P. Sabino et al., "The optical properties of mouse skin in the visible and near infrared spectral regions," J. Photochem. Photobiol. B 160, 72-78 (2016).

32. S. M. Marques et al., "Genetic background determines mouse strain differences in inflammatory angiogenesis," Microvasc. Res. 82(3), 246-252 (2011).

33. L. Azzi et al., "Gender differences in mouse skin morphology and specific effects of sex steroids and dehydroepiandrosterone," J. Invest. Dermatol. 124(1), 22-27 (2005).

34. K. Calabro et al., "Gender variations in the optical properties of skin in murine animal models," J. Biomed. Opt. 16(1), 011008 (2011).

35. L. S. Hansen et al., "The influence of the hair cycle on the thickness of mouse skin," Anat. Rec. 210(4), 569-573 (1984).

36. T. D. Dodig et al., "Development of the tight-skin phenotype in immune-deficient mice," Arthritis Rheum. 44(3), 723-727 (2001).

37. T. Durduran et al., "Diffuse optics for tissue monitoring and tomography," Rep. Prog. Phys. 73(7), 076701 (2010).

38. M. A. Ansari et al., "Diffuse optical tomography: image reconstruction and verification," J. Lasers Med. Sci. 5(1), 13-18 (2014).
39. F. Ayers et al., "Fabrication and characterization of silicone-based tissue phantoms with tunable optical properties in the visible and near infrared domain," Proc. SPIE 6870, 687007 (2008).

40. R. B. Saager et al., "Multilayer silicone phantoms for the evaluation of quantitative optical techniques in skin imaging," Proc. SPIE 7567, 756706 (2010).

41. G. J. Greening et al., "Characterization of thin poly(dimethylsiloxane)based tissue-simulating phantoms with tunable reduced scattering and absorption coefficients at visible and near-infrared wavelengths," J. Biomed. Opt. 19(11), 115002 (2014).

42. K. Zhang and D. J. Waxman, "Impact of tumor vascularity on responsiveness to antiangiogenesis in a prostate cancer stem cell-derived tumor model," Mol. Cancer Ther. 12(5), 787-798 (2013).

43. U. Sunar et al., "Hemodynamic responses to antivascular therapy and ionizing radiation assessed by diffuse optical spectroscopies," Opt. Express 15(23), 15507-15516 (2007).

Syeda Tabassum is a PhD candidate in the Department of Electrical and Computer Engineering at Boston University. She conducts research in the Biomedical Optical Technologies Lab under the mentorship of Dr. Darren Roblyer.

Vivian Pera received her $A B$ degree in physics from Harvard University, and her MS and PhD degrees in electrical engineering from Tufts University and Northeastern University, respectively. She is a postdoctoral research associate in the Department of Biomedical Engineering at Boston University. Before returning to school to pursue a doctoral degree in biomedical optics, she worked at MIT Lincoln Laboratory developing adaptive signal processing algorithms for sonar and radar applications.

Gage Greening is a PhD candidate and NSF graduate research fellow in the Department of Biomedical Engineering at the University of Arkansas. He conducts research in the Translational Biophotonics and Imaging Laboratory under the mentorship of Dr. Timothy Muldoon.

Timothy J. Muldoon received his PhD from Rice University in 2009 and MD degree from the Baylor College of Medicine in 2010 and is now an associate professor of biomedical engineering at the University of Arkansas.

Darren Roblyer received his BS degree in biomedical engineering from Johns Hopkins University in 2004, and his PhD in bioengineering from Rice University in 2009. He did his postdoctoral work at the Beckman Laser Institute, University of California, Irvine. He is an assistant professor of biomedical engineering at Boston University. $\mathrm{He}$ is a senior member of SPIE. 